\title{
Educação Sexual e Reprodutiva para Adolescentes na Atenção Primária: uma Revisão Narrativa
}

\section{Sexual and Reproductive Education for Teens in Primary Care: a Narrative Review}

\author{
Mirieli Louveira de Figueiredo
}

Universidade para o Desenvolvimento do Estado e da Região do Pantanal. MS, Brasil.

E-mail: miri_miroca@live.com

\begin{abstract}
Resumo
As altas taxas de gravidez na adolescência e de casos de infecções sexualmente transmissíveis (IST) se tornaram os temas centrais de importantes debates em grande parte dos países, nas últimas décadas. De acordo com o Sinasc, o número de crianças nascidas de mães adolescentes representou 18\% dos 3 milhões de nascidos vivos em 2015 no Brasil. Segundo a Organização Mundial da Saúde (OMS), em 2016, surgiu mais de 1 milhão de novos casos de IST entre pessoas com idade de 15 a 49 anos. Esses dados evidenciam as lacunas existentes na aplicação de uma importante ferramenta de prevenção e promoção da saúde dos adolescentes: a educação em saúde. Por meio deste trabalho se pretende abordar as implicações da educação em saúde na sensibilização do adolescente em relação à saúde sexual e reprodutiva. Foi realizada uma revisão narrativa sendo utilizados artigos encontrados na base de dados SciELO (Scientific Electronic Library Online), além de cadernos de Atenção Básica do Ministério da Saúde. Foram analisados 30 artigos sobre o tema, em língua portuguesa e com datas de publicação no período de 2009 a 2018. Nota-se que ainda há um longo caminho a ser percorrido para que a educação sexual e reprodutiva seja de fato eficaz e integral durante o processo de adolescer. Problemas como a falta de humanização e acolhimento, além da manutenção de estereótipos e visão não holística do adolescente são alguns dos obstáculos a serem enfrentados neste cenário. A enfermagem tem condições e o dever de contribuir para a melhora da situação de saúde dos adolescentes brasileiros.
\end{abstract}

Palavras-chave: Saúde do Adolescente. Enfermagem. Educação. Sexualidade. Reprodução.

\begin{abstract}
In recent decades, the highs fees of adolescence pregnancy and sexually transmitted infections (STI) cases became the main subjects of important debates in many countries. According to SINASC (Live Birth Information System), in Brazil, the number of children born from adolescent mothers represents $18 \%$ of the 3 million live births in 2015. Moreover, according to the World Health Organization (WHO) (2016), more than 1 million new cases of STI arise among people aged by 15 to 49 years old. These data highlight the flaws in the application of an important implement for adolescent's prevention and health promotion: health education. This study aims to board the implications of health education in the adolescent's sensitizing regarding sexual and reproductive health. A narrative review was accomplished through articles found in the SciELO (Scientific Electronic Library Online) database and periodicals, in addition to Ministry of Health Primary Care notebooks. Thirty articles, which did not evade the theme, in Portuguese language, and had the publication dates from 2009 to 2018 , were analyzed. It is noted that there's still a long journey so that sexual and reproductive education are effective and integral in the adolescence process. Problems such as humanization and lack of acceptance, in addition to stereotype maintenance and non-holistic view of the adolescents represent s some of the obstacles to be faced in this scenario. Nursing professionals have the conditions and the duty to contribute for the improvement of Brazilians adolescents' health situation.
\end{abstract}

Keywords: Adolescent's Health. Nursing. Education. Sexuality. Reproduction.

\section{Introdução}

A adolescência é um período complexo e de grandes mudanças que necessita de atenção especial no âmbito da saúde. Nesta fase, com o início da puberdade, há o desenvolvimento da sexualidade, característica importantíssima, presente desde os primórdios da vida. Durante a construção da sexualidade, os adolescentes podem ser movidos pela libido e pela curiosidade, em busca de autoconhecimento e de prazer, o que pode predispô-los a situações de risco (HOFFMANN; ZAMPIERI, 2009).

A educação sexual e reprodutiva é, ainda, um recurso pouco utilizado para o fomento da prevenção de gravidez precoce e infecções sexualmente transmissíveis (IST).
Segundo a Organização Mundial da Saúde (OMS), as médias, tanto de casos de gravidez na adolescência, quanto de IST, no Brasil, quando comparadas às de países mais desenvolvidos, são muito elevadas. Ademais, estudos demonstram que mesmo que os adolescentes tenham conhecimento acerca dos métodos de prevenção, menos da metade pratica relações sexuais de forma protegida (FREITAS; CARVALHO; ARAÚJO, 2017).

Outrossim, a prática de relações sexuais tem início cada vez mais precoce entre os adolescentes. A falta de conhecimento acerca dos métodos de prevenção, tanto de gravidez, quanto de IST, é um fato presente na vida da maior parte dessa população (MESQUITA et al., 2017). Sendo assim, quais as implicações da educação em saúde no desenvolvimento da saúde sexual e reprodutiva dos adolescentes? 
Dado o exposto, ao decorrer deste trabalho será retratada a importância da educação em saúde para o desenvolvimento saudável da saúde sexual e reprodutiva do adolescente.

\section{Desenvolvimento}

\subsection{Metodologia}

Estudo qualitativo de revisão narrativa, com busca exploratória na base de dados SciELO (Scientific Electronic Library Online) e em periódicos, além de cadernos de Atenção Básica do Ministério da Saúde. As palavras-chave utilizadas para a busca de artigos científicos foram: Educação Sexual e Reprodutiva; Atenção Primária; Enfermagem; Promoção da Saúde. Os critérios de inclusão foram: artigo em texto completo, abordar a saúde e/ou direitos do adolescente, estar em Língua Portuguesa, ter data de publicação no período de 2009 a 2018 (últimos 10 anos). Foram encontrados 580.000 artigos, dos quais 30 foram selecionados, além de boletins epidemiológicos e cadernos/manuais, sendo que estes se enquadravam em todos os critérios de inclusão, além de apresentarem datas de publicação mais recentes entre o contorno estabelecido. Os artigos excluídos apresentavam um ou mais critérios de exclusão, principalmente, em relação à língua e ao período de publicação, além de apresentarem informações repetidas encontradas em outros estudos mais recentes.

\subsection{Sexualidade e Reprodução na Adolescência}

A adolescência é uma fase na qual mudanças físicas e psicossociais significativas ocorrem, estando intimamente relacionada às vivências e valores adquiridos ao longo da vida. Essa fase de transição pode ser desenvolvida de maneira saudável se os adolescentes tiverem a devida atenção às suas demandas e dúvidas relacionadas a este processo (MARTINS; NETO, 2014).

No que tange a saúde do adolescente, a educação sexual e reprodutiva ainda se revela como um grande tabu entre a sociedade, portanto, discutir essa temática é fundamental, principalmente, no âmbito da atuação da enfermagem, que tem grande papel na prevenção e promoção da saúde (LOPES; ANJOS; PINHEIRO, 2009).

Segundo a Organização Pan-Americana da Saúde (OPAS/ OMS), a adolescência compreende o período entre 10 e 19 anos de idade, sendo esta uma fase importantíssima, com transição da infância para a vida adulta. Nesta fase, há o acontecimento de diversas mudanças, estando essas inerentes a vários fatores, como cultura, religião e condições socioeconômicas em que os adolescentes se encontram. Pode-se inferir que a religião é um dos aspectos que mais influenciam no desenvolvimento do comportamento sexual e reprodutivo dos adolescentes, pois essa estabelece regras e normas sociais que afetam as atitudes destes (AMARAL et al., 2017).

O aumento do desejo sexual, nesta fase, coincide com o aparecimento dos caracteres sexuais secundários, tendo- se uma grande influência vinda da alta atividade hormonal presente neste momento. Neste cenário se tem a sexualidade, aspecto fundamental na formação do ser como um todo, estando presente na vida humana desde o nascimento (EISENSTEIN, 2013).

É comum que o termo "sexualidade" seja entendido como a prática da relação sexual em si, entretanto, esta propriedade abrange aspectos bem maiores, como gênero, realização pessoal, papéis sociais e reprodução. Com a construção da sexualidade, o adolescente passa a descobrir características próprias, suas preferências e anseios. Dessa forma, notase que este é um quesito crucial na formação da identidade do adolescente (MAROLA; SANCHES; CARDOSO, 2011; EISENSTEIN, 2013).

Diante de tantas mudanças e dúvidas em relação à sexualidade, existe, ainda, uma questão - conhecida há muito tempo, mas que vem sendo debatida mais fortemente e amplamente somente nos últimos anos -, que é inerente e tem grande impacto na saúde sexual e reprodutiva de uma significativa parcela da população adolescente: a desigualdade de gênero (AMARAL et al., 2017).

A sexualidade feminina sempre foi um aspecto reprimido e subjugado pela sociedade machista e patriarcal, desde os primórdios da vida. Sendo assim, a vivência deste aspecto, para as meninas, acaba sendo muito mais complicada, pois as mesmas não têm seus direitos assegurados, estando, quase sempre, em uma relação de subordinação aos homens. Dessa forma, é preciso que as ações de educação em saúde sejam embasadas no princípio da equidade (AMARAL et al., 2017).

Após o afloramento da sexualidade, é comum que os jovens sejam movidos pela curiosidade nos momentos em que têm a possibilidade de vivenciar experiências sexuais. Isto pode predispô-los a uma série de riscos, como a aquisição de IST e a ocorrência de abusos. Outros fatores determinantes neste contexto são as condições socioeconômicas e a própria cor/etnia dos adolescentes, sendo comum - considerando-se o tremendo histórico de preconceito presente na história do Brasil - que os jovens negros e/ou indígenas, principalmente do gênero feminino, estejam mais vulneráveis às situações de risco (MAROLA; SANCHES; CARDOSO, 2011).

Além das IST e HIV/AIDS, a gravidez na adolescência se apresenta como um dos maiores temas de debates sociais e de saúde pública no Brasil. As taxas de gravidez entre esta faixa etária são alarmantes e, muitas vezes, estampam a ineficiência e insuficiência das ações de prevenção da saúde voltadas a esse público. A ocorrência de uma gestação nesta fase modifica todos os setores e papéis já estabelecidos na vida da adolescente, pois a mesma precisa lidar com mudanças e exigências familiares e sociais, além das diversas mudanças físicas decorrentes da gestação (NUNES, 2010).

Segundo Berquó, Garcia e Lima (2012), a gravidez na adolescência está diretamente ligada ao nível de escolaridade e status econômico em que os adolescentes se encontram, 
portanto, quanto menor o favorecimento econômico e a escolaridade destes, menor o percentual de utilização de métodos contraceptivos.

Analisando-se os estudos sobre o tema, pode-se inferir que a precocidade da iniciação sexual, o desconhecimento e a falta de acesso, em relação aos métodos contraceptivos, são os principais fatores predisponentes à gravidez, sendo comum a utilização errônea de pílulas anticoncepcionais e anticoncepcionais de emergência ("pílula do dia seguinte"), além da persistência do uso isolado do preservativo masculino, em detrimento do feminino (NUNES, 2010).

Para muitos autores, a maternidade na adolescência se configura como fator determinante no desenvolvimento socioeconômico dos jovens, estando esta, muitas vezes, ligada ao abandono escolar e, consequentemente, à escassez de oportunidade de colocação no mercado de trabalho. Ademais, ressalta-se que as gravidezes neste período possuem grande potencial de se tornarem de risco (WIESE; SALDANHA, 2011). De acordo com o Ministério da Saúde (2017), dados do Sinasc demonstram que $66 \%$ das gravidezes nesta faixa etária são indesejadas.

Em contrapartida, cabe salientar que estudos de alguns autores, como Rodrigues, Barros e Soares (2016), permitem que se perceba a extrema homogeneização de concepçõesnegativas acerca da gravidez na adolescência, sendo que a maioria dos estudos encontrados na literatura possui um olhar estigmatizante e generalista acerca do assunto, sem o reconhecimento de que muitas adolescentes desejam a gravidez e se sentem realizadas com esta.

Apesar de, teoricamente, afetar ambos os lados, a realidade da grande maioria dos casos de gravidez na adolescência demonstra, mais uma vez, o caráter machista da sociedade, sendo comum que mães adolescentes cuidem de seus filhos por si sós ou recebam ajuda de familiares para tal, sem a participação paterna devida. Tais problemas apontam a clara necessidade de elaboração de políticas mais específicas e equitativas voltadas ao público adolescente feminino (BERQUÓ; GRACIA; LIMA, 2012).

\subsection{O Adolescente na Conjuntura das Políticas Públicas Brasileiras}

O público adolescente representa uma grande parcela da população total do Brasil, tendo este, então, uma expressiva significância demográfica para o país. Nesse contexto, a discussão e criação de políticas públicas específicas voltadas a esses indivíduos se apresentou como uma necessidade para a garantia de direitos e redução de vulnerabilidades, as quais os adolescentes estão expostos, isso se deve, principalmente, às pressões feitas por movimentos sociais organizados, como o feminista, LGBT e de Juventude (MARTINS; NETO, 2014).

Segundo Jager et al. (2014), apenas a partir 1986, o Estado, através do Ministério da Saúde, passou a integrar a assistência primária à saúde da população jovem e adolescente, tendo como primeiras ações programas com foco na prevenção de doenças sexualmente transmissíveis. O Programa Saúde do Adolescente (PROSAD), criado em 1989, apresenta-se como a primeira política criada, especificamente, para o público adolescente no Brasil.

O PROSAD teve como foco atender às demandas dos adolescentes, abrangendo temas como gravidez precoce, IST, além do álcool e outras drogas. Entretanto, ao longo de sua aplicação, o mesmo se mostrou desintegrado e não universal, pois não possibilitava aos adolescentes a autonomia necessária para que os mesmos se tornassem agentes ativos em seus próprios processos de promoção da saúde. Sendo assim, o programa foi reorientado, posteriormente, à efetivação da Política Nacional de Atenção Integral à Saúde de Adolescentes (PNAISA) em 2008 (JAGER et al., 2014).

Para Eduardo e Egry (2009), o Estatuto da Criança e do Adolescente - ECA (BRASIL, 1990), Lei no 8.069 de 13 de julho de 1990, caracteriza-se como o mais importante documento norteador das políticas e programas voltados ao público adolescente e infantil. Esta lei estabelece e regulamenta os direitos das crianças e dos adolescentes brasileiros, além de discorrer sobre os papéis de diversos agentes sociais - como o Estado, a família, a comunidade e as escolas - para a garantia da proteção integral dos adolescentes.

Fonseca et al. (2013) destacam que a criação do ECA se constitui como um meio de incluir crianças e adolescentes que vivem em situação de vulnerabilidade social nas políticas públicas de saúde. Ponderar acerca das vulnerabilidades as quais os adolescentes brasileiros estão expostos se torna, portanto, fundamental para a criação de políticas universais e integrais. A percepção do estado de saúde como o bem-estar físico, mental e social - como definido pela OMS -, passou, então, a ser preconizada na elaboração das políticas futuras.

De acordo com Horta e Sena (2010), a partir de 1999 houve aumento significativo das políticas referentes à temática dos adolescentes e jovens, quando comparada ao cenário dos anos anteriores. É após este período que as políticas começam a ganhar espaço, à medida que surgem projetos e programas que buscam enfrentar dois grandes problemas aos quais os jovens estão mais expostos: a violência e o desemprego.

A Política Nacional de Atenção Integral à Saúde de Adolescentes, criada em 2004 e aprimorada em 2008, apresenta-se como um grande avanço no âmbito da assistência à saúde dos adolescentes. Esta política propôs um novo olhar ao processo de adolescer, no qual - com base na Constituição Federal (1988) e no Estatuto da Criança e do Adolescente (1990)-, reconhece os adolescentes como pessoas independentes e de direito, estabelecendo diretrizes e estratégias para o atendimento de saúde voltado a este público (JAGER et al., 2014).

Em relação à condição socioeconômica, nota-se que esta se apresenta como importante fator na determinação do desenvolvimento saudável da adolescência, estando inerente à construção da cidadania. Nesse contexto, o Governo Federal criou, em 2003, o Programa Bolsa Família 
(PBF), visando beneficiar as famílias brasileiras carentes e, consequentemente, as crianças e os adolescentes. O programa se baseia na transferência de renda às famílias em situação de pobreza ou extrema pobreza e possibilita aos adolescentes maior acesso aos serviços de saúde, informação, além de métodos de prevenção (SCHAEFER et al., 2018; RAPOSO, 2009).

No que tange ao princípio da universalidade, uma questão muito importante, que ainda não possui a visibilidade devida, é a existência de um grupo entre a população adolescente que merece a garantia de direitos e assistência, tanto quanto qualquer outro: os adolescentes em conflito com a lei. Segundo Cruz et al. (2010), estes jovens, muitas vezes negligenciados através da grande exclusão social que sofrem, possuem os mesmos direitos - garantidos pelo ECA -, que os adolescentes não infratores.

A elaboração de políticas públicas integrais - de curto, médio e longo prazo - mais efetivas, que possibilitem aos jovens o protagonismo que lhes é de direito, e a aplicação destas na prática por parte dos profissionais são medidas fundamentais para a promoção da saúde dos adolescentes (MOTA, 2014; MOREIRA et al., 2015).

\subsection{A Enfermagem na Educação em Saúde Sexual e Reprodutiva do Público Adolescente}

Considerando o âmbito da sexualidade e da reprodução, quando se trata de adolescentes, muitos profissionais da área da saúde acabam promovendo a ideia de que esta população é sempre irresponsável, inexperiente e imatura, o que acaba afastando os jovens das ações educativas oferecidas pelas unidades de saúde, pois os mesmos se sentem repreendidos e julgados, sem a possibilidade de criação de vínculo com os profissionais ou de atuação ativa no processo de adquirir conhecimento (SCHAEFER et al., 2018).

Problemas como estes comprometem os objetivos de prevenção e de promoção da saúde, pretendidos na criação das Unidades de Saúde da Família (USF), em 1994. As USF representam a principal porta de entrada da população para acesso aos serviços de saúde, estando estas também em âmbito de atenção com papel protagonista no cuidado à saúde dos adolescentes, a atenção primária (SENA FILHA; CASTANHA, 2014).

Segundo Tôrres, Nascimento e Alchieri (2013), a frequência com que os adolescentes procuram os serviços de saúde ainda é insatisfatória. Os atendimentos odontológicos e médicos são os mais procurados, e apenas quando já há conhecimento de doenças instaladas. Dessa forma, as unidades de saúde são vistas apenas como prestadoras de serviços assistencialistas, e não de educação. Isso faz com que outros ambientes sejam utilizados para a realização de ações de promoção da saúde, como escolas e universidades.

O profissional de enfermagem, atuante na atenção primária, tem a possibilidade de promover a saúde sexual e reprodutiva dos adolescentes através de ações de educação em saúde nas comunidades, pois pode intervir junto à população, coordenando estratégias específicas. Para tal, é necessário que o profissional leve em consideração os diversos fatores que influenciam a sexualidade e reprodução do adolescente, como crenças religiosas, tabus, cultura e experiências sociais (SOUSA; COELHO, 2014).

De acordo com Alves e Brandão (2009), ainda que os programas e diretrizes tenham sidos aprimorados ao longo dos anos, a realidade da vida sexual e reprodutiva da grande maioria dos adolescentes ainda é cercada de mitos, de tabus e de atitudes que colocam sua saúde em risco, como: utilização do preservativo apenas em relações esporádicas, prática do "coito interrompido", além da falta de conhecimento em relação à utilização da pílula anticoncepcional e à "pílula do dia seguinte".

A aplicação das diretrizes das políticas públicas na prática tem demonstrado grandes falhas, seja por problemas nas próprias políticas, e/ou problemas em relação às atitudes dos profissionais - que também necessitam de capacitação permanente e continuada - como também pela falta de recursos humanos e materiais (BRASIL, 2010).

As ações de educação em saúde visam cada vez mais a autonomia dos participantes, estimulando-os a tomarem frente na tomada de decisões, de resolução de problemas e de análise crítica de situações. A enfermagem vem aprimorando os recursos que podem ser utilizados para este fim, inserindo novas tecnologias e dinâmicas, como jogos educativos, oficinas e rodas de conversa, que estimulam a conscientização e participação ativa dos usuários (KOERICH et al., 2015).

Os estudos apresentam unanimidade em relação à importância da escola nestas ações. Este é o principal meio utilizado para fomento da educação em saúde voltada aos adolescentes, já que é um ambiente com grande concentração de jovens e no qual os mesmos passam a maior parte de seus dias (MOREIRA et al., 2015).

Em 2003, o Ministério da Saúde criou o Projeto Saúde e Prevenção nas Escolas (PSE) - visando diminuição dos casos de infecção por IST e redução dos índices de evasão escolar causada pela gravidez precoce -, sendo este um grande marco na articulação saúde-educação. Os enfermeiros integrantes das Equipes de Saúde da Família são uns dos principais agentes atuantes nesta integração (MOREIRA et al., 2015).

A maioria dos estudos evidencia o escasso conhecimento acerca da anatomia e fisiologia do corpo, principalmente feminino, sendo este bastante limitado entre o público adolescente, tendo-se ainda a masturbação como um grande tabu para as meninas. Além disso, é notável o quanto a realização de atividades em grupo gera respostas positivas entre os jovens, já que os mesmos se sentem acolhidos e têm a possibilidade de criar vínculos de confiança com os colegas (BRASIL, 2016).

Em relação ao conhecimento acerca dos métodos de prevenção, Freitas, Carvalho e Araújo (2017) demonstram em seu estudo que este se concentra apenas no preservativo 
masculino, sendo o método mais conhecido entre essa população. Nessa perspectiva, é comum o fato de os adolescentes não enxergarem suas famílias como fontes de informação, o que aponta a presença de lacunas no processo de educação em saúde, considerando-se que este deve englobar os familiares dos jovens também.

Souza, Morais e Oliveira (2015) abordaram em seu estudo como os Materiais Educativos Impressos (MEI), como cartazes, cartilhas e panfletos, influenciam no processo de educação em saúde sexual e reprodutiva, demonstrando que ainda existem diversos hiatos na articulação da sistematização teórico-metodológica com os materiais educativos, sendo que estes, apesar dos discursos contrários dos profissionais, na maioria das vezes não são utilizados como ferramentas de fomento à educação sexual e reprodutiva.

As ações educativas se tornam imprescindíveis, visto que, somente a partir da sensibilização do adolescente acerca da autonomia e da responsabilidade deste com seu próprio corpo, será possível que haja o desenvolvimento saudável de sua sexualidade e identidade. Os resultados dos estudos referentes à aplicação de métodos de educação, como oficinas e jogos, demonstram que os jovens adquirem grande quantidade de informações novas, além de terem mitos desmistificados durante as ações. Ademais, ressalta-se que a associação destes métodos a recursos visuais e aos MEI's seja a forma mais efetiva de se educar (MOREIRA et al., 2015).

É preciso salientar que as ações de educação em saúde se fazem necessárias não só no âmbito escolar, mas em todas as áreas cabíveis da comunidade, visto que a evasão escolar é fato presente na vida de muitos adolescentes. Portanto, os profissionais de enfermagem, atuantes na atenção primária, devem realizar a busca ativa desse público, ampliando os meios de chegada de informação. Dessa forma, tem-se o estímulo da autonomia e do autocuidado, com construção de uma não relação de dependência profissional, cabendo ao enfermeiro o provimento de oportunidades de participação do adolescente (MARTINS et al., 2011).

\section{Conclusão}

A atenção primária representa a porta de entrada dos usuários aos serviços de saúde e é o meio mais propício para a realização de ações preventivas, sendo o enfermeiro um dos principais atores neste cenário. É preciso que as estratégias de educação em saúde sejam atualizadas e elaboradas conforme as particularidades de cada comunidade, além de considerar vulnerabilidades relacionadas ao gênero, orientação sexual, etnia e cor. A busca ativa dos adolescentes é fundamental.

No que concerne à literatura disponível, percebe-se que maioria dos estudos aborda o tema de forma a caracterizálo como uma questão social e de saúde pública, sendo esses desenvolvidos, majoritariamente, por profissionais e/ou acadêmicos de cursos da área da saúde. Há uma boa quantidade de artigos com datas de publicações recentes, o que demonstra a importância do tema e a crescente busca por debatê-lo. .

\section{Referências}

ALVES, C.A.; BRANDÃO, E.R. Vulnerabilidades no uso de métodos contraceptivos entre adolescentes e jovens: interseções entre políticas públicas e atenção à saúde. Ciênc. Saúde Coletiva., v.14, n.2, p.661-670, 2009. doi: 10.1590/S141381232009000200035

AMARAL, A.M.S. et al. Adolescência, gênero e sexualidade: uma revisão integrativa. Rev. Enf. Cont., v.6, n.1, p.62-67, 2017. doi: 10.17267/2317-3378rec.v6i1.1114

BERQUÓ, E.; GARCIA, S.; LIMA, L. Reprodução na juventude: perfis sociodemográficos, comportamentais e reprodutivos na PNDS 2006. Rev. Saúde Pública, v.46, n.4, p.685-693, 2012. doi: 10.1590/S0034-89102012005000048

BRASIL. Lei no 8.069, de 13 de julho de 1990. Dispõe sobre o Estatuto da Criança e do Adolescente e dá outras providências. Diário Oficial [da] República Federativa do Brasil, Brasília, DF, 16 jul. 1990.

BRASIL. Ministério da Saúde. Boletim Epidemiológico de Hepatites Virais 2017. Disponível em: < http://www.aids. gov.br/pt-br/pub/2017/boletim-epidemiologico-de-hepatitesvirais-2017>. Acesso em: 20 set. 2018.

BRASIL. Boletim epidemiológico HIV/Aids 2017. Disponível em: $\quad<$ http://www.aids.gov.br/pt-br/pub/2017/boletimepidemiologico-hivaids-2017> . Acesso em: 20 mar. 2019.

BRASIL. Cuidando de adolescentes: orientações básicas para a saúde sexual e a saúde reprodutiva. Brasília: MS, 2016.

BRASIL. Diretrizes nacionais para a atenção integral à saúde de adolescentes e jovens na promoção, proteção e recuperação da saúde, Brasília: MS, 2010.

BRASIL. Gravidez na adolescência tem queda de $17 \%$ no Brasil. 2017. Disponível em: <http://www.saude.gov.br/noticias/ agencia-saude/28317-gravidez-na-adolescencia-tem-queda-de17-no-brasil>. Acesso em: 14 mar. 2019.

CRUZ, L.R. et al. Medidas socioeducativas em meio aberto no município de Santa Cruz do Sul/RS: entre as diretrizes legais e as políticas sociais públicas. Pesq. Prát. Psicossoc., v.5, n.1, 2010.

EDUARDO, L.P.; EGRY, E.Y. Estatuto da Criança e do Adolescente: a visão dos trabalhadores sobre sua prática. Rev. Esc. Enf. USP, v.44, n.1, p.18-24, 2010. doi: 10.1590/S008062342010000100003

EISENSTEIN, E. Desenvolvimento da sexualidade da geração digital. Adolesc. Saúde, v.10, supl.1, p.61-71, 2013.

FONSECA, F.F. et al. As vulnerabilidades na infância e adolescência e as políticas públicas brasileiras de intervenção. Rev. Paul. Pediatria, v.31, n.2, p.258-264, 2013. doi: 10.1590/ S0103-05822013000200019

FREITAS, N.O.; CARVALHO, K.E.G.; ARAÚJO, E.C. Estratégia de educação em saúde para um grupo de adolescentes do Recife. Rev. Adolesc. Saúde, v.14, n.1, p.29-36, 2017.

HOFFMANN, A.C.O.S.; ZAMPIERI, M.F.M. A atuação do profissional da enfermagem na socialização de conhecimentos sobre sexualidade na adolescência. Rev. Saúde Públ, v.2, n.1, 2009.

HORTA, N.C.; SENA, R.R. Abordagem ao adolescente e ao jovem nas políticas públicas de saúde no Brasil: um estudo de revisão. Physis, v.20, n.2, p.475-495, 2010. doi: 10.1590/S010373312010000200008 
JAGER, M.E. et al. O adolescente no contexto da saúde pública brasileira: reflexões sobre o PROSAD. Psicol. Estud., v.19, n.2, p.211-221, 2014. doi: 10.1590/1413-737221567004

KOERICH, C. et al. Gestão do cuidado de enfermagem ao adolescente que vive com HIV/AIDS. Esc. Anna Nery, p. 115123, 2015. doi: 10.5935/1414-8145.20150016

LOPES, E.M.; ANJOS, S.J.S.B.; PINHEIRO, A.K.B. Tendência das ações de educação em saúde realizadas por enfermeiros no Brasil. Rev. Enferm. UERJ., v.17, n.2, p.273-277, 2009.

MAROLA, C.A.G.; SANCHES, C.S.M.; CARDOSO, L.M. Formação de conceitos em sexualidade na adolescência e suas influências. Psicol. Educ., n.33, p.95-118, 2011.

MARTINS, C.B.G. et al. Oficina sobre sexualidade na adolescência: uma experiência. Rev. Mineira Enferm., v.15, n.4, p.573-578, 2011.

MARTINS, N.R.M.; NETO, J.M. Adolescente, esse ser em transformação. In: PARANÁ. Secretaria de Estado da Educação. Superintendência de Educação. Os desafios da escola pública paranaense na perspectiva do professor PDE. Curitiba: SEED/ PR, v.1, p.1-19, 2014.

MESQUITA, J.S. et al. Fatores de risco e de proteção entre adolescentes em relação às DST/HIV/AIDS. Rev. Enferm. UFPE, v.11, n.3, p.1227-1233, 2017. doi: 10.5205/reuol.10544-93905-1RV.1103201713

MOREIRA, W.C. et al. Ações educativas do enfermeiro na promoção da saúde sexual e reprodutiva do adolescente. Rev. Interdisc., v.8, n.3, p.213-220, 2015.

MOTA, A.A.S. Ações de prevenção voltadas para a saúde sexual e reprodutiva na adolescência no município de Três Marias Minas Gerais. Sete Lagoas: Universidade Federal de Minas Gerais, 2014.

NUNES, S.A. Esperando o futuro: a maternidade na adolescência. Physis, v.22, n.1, p.53-75, 2012. doi: 10.1590/ S0103-73312012000100004

OPAS - Organização Pan-Americana da Saúde. Organização Mundial da Saúde. Determinantes sociais e riscos para a saúde, doenças crônicas não transmissíveis e saúde mental. Folha informativa - Saúde mental dos adolescentes. 2018. Disponível em: https://www.paho.org/bra/index.php?option=com content $\&$ view $=$ article \&id=5779:folha-informativa-saude-mental-dosadolescentes\&Itemid=839> . Acesso em: 15 mar. 2019.

RAPOSO, C. A política de atenção integral à saúde do adolescente e jovem: uma perspectiva de garantia de direito à saúde?. Rev. Fac. Serv. Soc. Univ. Estado Rio Janeiro, v.6, n.23, 2009. doi: 10.12957/rep.2009.450

RODRIGUES, A.R.S.; BARROS, W.M.; SOARES, P.D.F.L. Reincidência da gravidez na adolescência: percepções das adolescentes. Enferm. Foco, v.7, n.3/4, p.66-70, 2016. doi: 10.21675/2357-707X.2016.v7.n3/4.945

SCHAEFER, R. et al. Políticas de saúde de adolescentes e jovens no contexto luso-brasileiro: especificidades e aproximações. Ciênc. Saúde Coletiva, v.23, n.9, p.2849-2858, 2018. doi: 10.1590/141381232018239.11202018

SENA FILHA, V.M.; CASTANHA, A.R. Profissionais de unidades de saúde e a gravidez na adolescência. Psicol. Soc., v.26, p.79-88, 2014. doi: 10.1590/S0102-71822014000500009

SOUSA, M.G.; COELHO, M.M.F. Contando bem, que mal tem? Construção de tecnologia educativa sobre sexualidade para promoção da saúde com adolescentes. Rev. Diálogos Acad., v.3, n.2, 2014.

SOUZA, L.M.; MORAIS, R.L.G.L.; OLIVEIRA, J.S. Direitos sexuais e reprodutivos: influências dos materiais educativos impressos no processo de educação em sexualidade. Saúde Debate, v.39, n.106, p.683-693, 2015. doi: 10.1590/0103110420151060003010

SOUZA, V. Adolescentes em cena: uma proposta educativa no campo da saúde sexual e reprodutiva. Rev. Esc. Enferm. USP, v.45, p.1716-1721, 2011. doi: 10.1590/S0080-62342011000800014

TÔRRES, T.R.F.; NASCIMENTO, E.G.C.; ALCHIERI, J.C. $\mathrm{O}$ cuidado de enfermagem na saúde sexual e reprodutiva dos adolescentes. Adolesc.,. Saúde, v.10, supl.1, 2013.

WIESE, I.R.B.; SALDANHA, A.A.W. Vulnerabilidade dos adolescentes às DST/AIDS: ainda uma questão de gênero?. Psic., Saúde Doenças, v.12, n.1, p.105-118, 2011. 